\title{
OPERATING POINT SELECTION AND BURN STABILTY CONTROL FOR THE INTERNATIONAL THERMONUCLEAR EXPERIMENTAL REACTOR
}

\author{
S.W. Haney and LJ. Perkins \\ Lawrence Livermore National Laboratory \\ P.O. Box B08, L-644 \\ Livermore, California 94550
}

UCRL--101947

DE 90002219

\begin{abstract}
The selection and burn stability control of near-ignited operating points for the International Thermonuclear Experimental Reactor (ITER) are considered. It is suggested that high density $\left(\left\langle n_{e}\right\rangle-1-2 \times 10^{20} \mathrm{~m}^{-3}\right)$, low temperature $\left(<T_{e}>_{n}-6-10 \mathrm{KeV}\right)$ operating points are preferred from considerations of proximity to magnetohydrodynamic (MHD) stability limits, divertor operation, and fueling even though those points are intrinsically thermally unstable. Results from simple 0-D transport simulations suggest that these operating points can be effectively stabilized at $Q>50$ if neutral beam feedback based on total neutron flux measurements is employed.
\end{abstract}

\section{Introduction}

One of the missions of ITER is the demonstration of the capability for long puise, ignited (or high- $Q$ ) operation at fusion power levels sufficient to ensure that the heating power required is insignificant compared with the alpha particle power produced [1]. The success of this mission depends (1) on the ability to obtain plasma conditions yielding power and particle balance equilibria consistent with constraints imposed by MHD stability, divertor operation, and fueling and (2) on the ability to devise control strategies for maintaining the plasma conditions for the desired pulse length.

In this paper, we will address the questions of operating point selection and control for the ITER physics phase machine which is characterized by major radius $R_{0}=6 \mathrm{~m}$, minor radius $a=2.15 \mathrm{~m}$, elongation $\mathrm{ks}=2.22$ (at the separatrix), elongation $K=1.96$ (at the 95\% flux surface), triangularity $\delta=0.6$ (at the separatrix), plasma current $\mathrm{I}_{p}=22 \mathrm{MA}$, and vacuum toroidal field $B_{0}=4.85 \mathrm{~T}$.

\section{N.odel}

Our analysis consists of simulations of the timedependent, $0-D$ transport equations for fuel tons, thermalized alpha particles, and electron/ion energy density in the presence of fueling and a time-varying neutral beam heating input. These equations are derived by averaging the two-fluid transport equations over the plasma volune (assumed to be elliptical) under the assumption that the density and temperature profiles are of the form

$$
n, T=n_{0}, T_{0}\left(1-\frac{\left(R-R_{0}\right)^{2}}{a^{2}}-\frac{z^{2}}{k^{2} a^{2}}\right)^{\alpha_{n, T} T}
$$

where $\alpha_{n}=0.5$ and $\alpha_{T}=1.0$ for all time. The $x_{n, T}$ choices reflect the belief that central fueling might not be achievable in ITER [2]. The $0-D$ approach was chosen over more accurate and elaborate methods such as 1.5-D transport to allow consideration of a relatively wide range of plasma conditions and control strategies.

We model L-mode energy confinement using the new ITER offset-linear scaling developed by Yushmanov, et al [3]

$$
\begin{gathered}
\tau_{E}(\mathrm{~L} \text {-mode })=0.64 M^{0.2} I_{p}^{0.8} R_{0}^{1.6} a^{0.6} \kappa_{S}^{0.2} \bar{n}_{e}^{0.6} B_{0}^{0.35} / P+ \\
0.04 M^{0.5} I_{p}^{0.5} R_{0}^{0.3} a^{0.8} \kappa_{S}^{0.6}
\end{gathered}
$$

where $M$ is the average isotopic mass, $\tilde{n}_{e}$ is the lineaveraged electron density, and $P$ is the net plasma heating power (alpha power + neutral beam power + ohmic power - radiation power). We further assume that $\mathrm{H}$ mode operation will be possible so $\tau_{E}=H \tau_{E}$ (L-mode) where $H$ is the L-mode enhancement factor. Fuei ion particle confinement times are taken to have the scaling $\tau_{p}=3 \tau_{E}$ while the net thermal alpha particle confinement time (which actually depends sensitively on edge effects such as recycling and pumping) is simply chosen to yield a specific equilibrium concentration of thermal alpha particles $c_{\alpha}$.

\section{Operating Point Selection}

We address ITER operating point selection using a convenient graphical representation for reactor performance developed by Houlberg, et al [4]. This method consists of plotting contours of the neutral beam power required for power balance equilibrium $P_{N B, E_{q}}$ as a function of the density-weighted volume-averaged electron temperature $\left\langle T_{E}\right\rangle_{n}$ and the volume-averaged electron density $\left\langle\pi_{e}\right\rangle$. Each point in these plots corresponding to a non-negative value of $P_{N B}, E_{q}$ represents a physical solution to the steady state $0-D$ tiansport equations and, hence, is a potential operating point.

A set of these contours (constructed assuming $c_{\alpha}=5 \%$ and $H=2.3$ ) for the ITER physics phase machine is shown in Fig. 1. Also shown are contours describing those $\left\langle n_{e}\right\rangle-$ $\left\langle T_{e}\right\rangle_{n}$ combinations yielding an average neutron wall loading $P_{w}=1 \mathrm{MW} / \mathrm{m}^{2}$ (corresponding to a fusion power $\left.P_{f}=\mathrm{IO} 82 \mathrm{MW}\right)$ and those $\left\langle n_{e}\right\rangle-\left\langle r_{e}\right\rangle_{n}$ combinations. characterized by beta values at the MHD stability limit (assumed to be represented by $\beta(\%)<2.5 l_{p} / a B_{0}[2$ !). We $=+\infty$ select the ITER operating point from this $1 \mathrm{MW} / \mathrm{m}^{2}$ contour because parametric studies [5] nave shown that $P_{t}$ - $1 \mathrm{MW} / \mathrm{m}^{2}$ and $H=2.3$ are necessary to ensure high- $Q-$ $\therefore$ 10 Or 


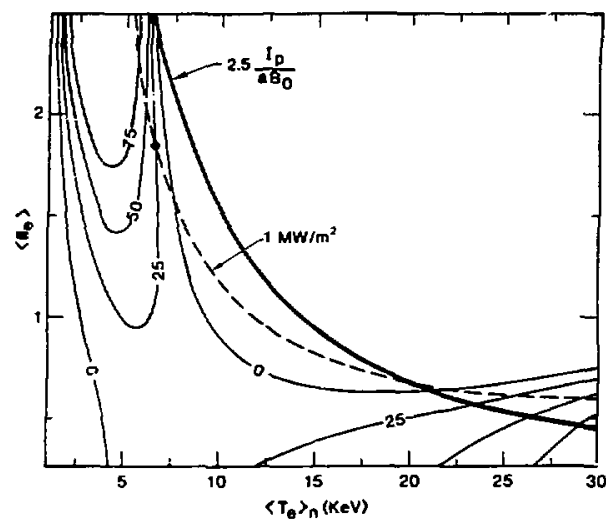

Figure 1: Contours of equilibrium neutral beam power $P_{N B, E_{q}}$ as a function of density $\left\langle n_{e}\right\rangle$ and temperature $\left\langle T_{e}\right\rangle_{n}$ with alpha particle fraction $c_{\alpha}=5 \%$ and L-mode enhancement $H=2.3$. Also shown are contours yielding a wall loading $P_{w}=1 \mathrm{MW} / \mathrm{m}^{2}$ and $a p(s)=2.5 I_{p} / a B_{0}$. A potential ITER operating point is labeled with a $\bullet$.

uperation of the ITER physics phase machine at end of burn conditions (where $c_{\alpha}$ may approach 10\%). We assume that both $P_{w}$ and $H$ ramain fixed for the entire burn.

Notice from Fig. I that there are two ignited equilibrium points (i.e., places where the $P_{w}=1 \mathrm{MW} / \mathrm{m}^{2}$ contour interserts the $P_{N B}, \varepsilon_{q}=0$ contour): one characterized by high density, low temperature and another characterized by low density, high temperature. There are also two regions of sub-ignited $\left(P_{N B, E_{q}}>0\right)$ operation. From the point of view of power/particie balance, all of these equilibrium points are equally acceptable. However, it can be argued that the high density, low temperature equilibrium points might be the most desirable for ITER high-Q operation. In particular, the low density equilibrium points are typically characterized by beta values close to the MHD stability limit and, in fact, for the conditions in Fig. 1, we see that the low density points actually exceed the beta limit (and, hence, are not acceptable). Additionally, higher densities tend to reduce the energies of particles impacting on the divertor plates (thus reducing sputtering) and lower temperatures tend to reduce the cffect of fuel peliet ablation (thus allowing bet:er pellet penetration).

Although favored from MHD stability, divertor, and fueling considerations, the high density equilibrium points suffer from a potentially serious drawback: they are susceptible to thermal instabilities (i.e., uncontrolled increases or decreases in temperature). To see this, consider the point labcled with a $*$ in Fig. 1 . If the ter-perature increases from its equilibrium value, perhaps due to a random fluctuation, the plasma enters a region in
$<n_{e}>-<T_{e}$ in space where the neutral beam power required to maintain equilibrium is less than at the original operating point. This means that power sources exceed losses and the temperature must continue to increase even more. A similar argument can be made ragarding a decrease in the temperature. On the basis of this discussion, one can reason that an operating point is unstable to temperature perturbations if [6]

$$
\frac{\partial P_{N B, E_{q}}}{\partial<T_{e}>_{n}}<0
$$

Applying Eq. (3) to the contcurs in Fig. 1 confirms that the high density, low temperature points represent thermally unstable equilibria. If IIER is to operate at these points, methods for controlling the thermal instabilities must be demonstrated. This is the topic of the next section.

\section{Operating Point Control}

Figure 2 shows a simulation of a typical ITER thermal instability. In this case, the electron and ion temperatures associated with the equilibrium point labeled with the ? in Fig. 1 (where $P_{N B, E}=25 \mathrm{MW}$ ) are instantaneously in reased by an amount $\Delta T_{e, i}=+0.36 \mathrm{KeV}$ (which yields a fractional change in the neutron fux $\phi_{N}$ of $\Delta \phi_{N} / \phi_{N}=$ $+15 \%$ ). Then, the plasma is allowed to evolve freely (with $H$ and $P_{N B}, E_{q}$ held fixed). We see from Fig. 2a (dashed curve) that the fusion power rapidly increases past 1500 MW over a 2 sec period. Subsequently, the MHD beta limit is exceeded, presumably resulting in a hard disruption at a high fusion power level.

Negative excursions must also be considered. As Fig. 3a shows (dashed curve), assuming $\Delta T_{e, j}=-0.38 \mathrm{KeV}$ (which yields $\Delta \phi_{N} / \phi_{N}=-15 \%$; leads to a rapid decrease in the fusion power level. It is likely that this instability will also result in a disruption due to exceeding a ciensity limit which, for JTER, currently iakes the form [2]

$$
P_{\text {red }} \text { (within the } q=2 \mathrm{~s} \cdot \mathrm{ufacc} \text { ) }<0.7 P
$$

where $P_{\text {rad }}$ is the total sadiation power, $q$ is the MHD safety factor, and $P$ is the total plasma heating power. In the simulation shown in Fig. 3, Eq. (4) is violated within $4 \mathrm{sec}$ at the point when the fusion power drops to approximately $720 \mathrm{MW}$.

Since the time required for the thermal instability to cause either beta or density limit disruptions is short compared to the expected ITER burn length $(\sim 200 \mathrm{sec})$, it is necessary to consider metnods for actively controlling the instability. One of the more credible methods is control by modulated auxiliary heating [7]. In this sclieme, the plasma is operated slightly sub-ignited, but at high $Q$. Then, in response to an increase in the plasma temperature or fusion power, some or all of the heating power is ternporarily removed, hopefully returning the plasma to equilibrium conditions. Similarly, when the temperature or fusion power decreases, additional power is applied. Modulated auxiliary heating has been chosen as the baseline control method for the proposed Compact 
Ignition Tokamak (CIT) [8]. Other approaches to ITER burn control are analyzed by Mandrekas and Stacey [9].

We propose using modulation of the neutral beam power based on measurements of total neutron flux as the primary control method for ITER. In particular, we employ the simple proportional feedback law

$$
P_{N B}=P_{N B, E q}-G_{\phi}\left(\frac{\phi_{N}}{\phi_{N, E_{q}}}-1\right)
$$

where $G$, is the feedback gain (taken to be $1000 \mathrm{MW}$ for this study, indicating essentially instantaneous power response) and $\phi_{N, E_{q}}$ is the equilibrium neutron flux. The applied neutral beam power $P_{N B}$ is further constrained by
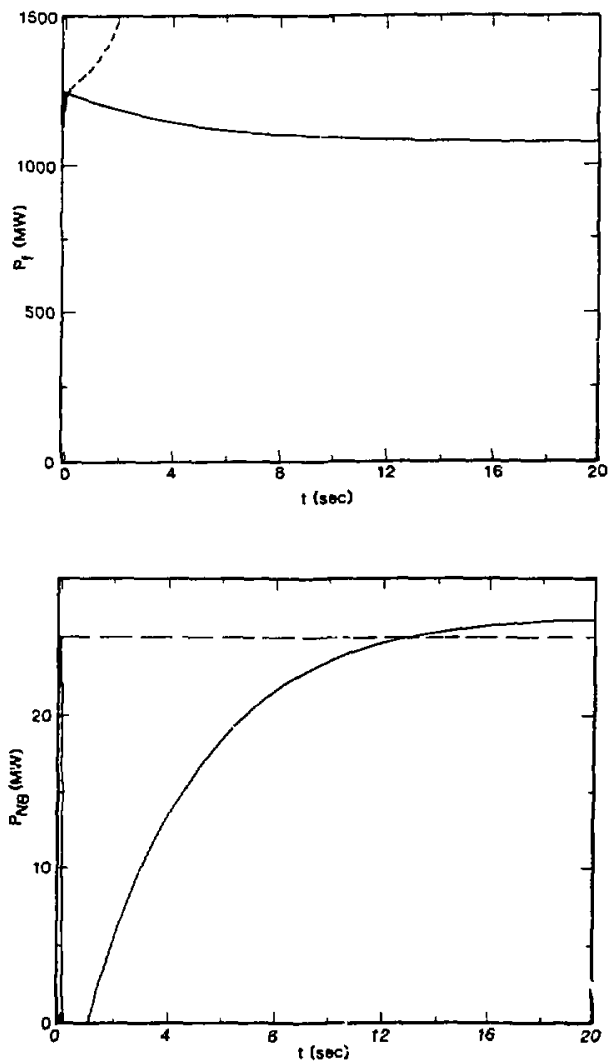

Figure 2: Simulations of an ITER thermal instability due to $\Delta T_{e, i}=; 0.36 \mathrm{KeV}$ (or $\Delta \phi_{N} / \phi_{N}=+15 \%$ ) pertiurbation of the equilibrium point labelod with a - in Fig. 1 . Shown are plots of (a) fusion power $P_{f}$ and (b) neutral beam power $P_{N B}$ as function of time for cases with (solid line) and without (dashed line) feedback. the inequality $0 \leq P_{N B} \leq P_{N B, m a x}$ where $P_{N B, \max }-75 \mathrm{MW}$ is the expected maximum neutral beam power capacity for ITER.

The choice of total neutron nux as the control signal is motivated by the observation that small changes in temperature lead to larger, and easier to measure, changes in neutron flux. For instance, measuring the $\Delta T_{c}=$ $+0.36 \mathrm{KeV}$ perturkation from the simulation in Fig. 2 might be difficult using current temperature diagnostic techniques sucin as electron cyclotron emission. However, the resulting $15 \%$ fractional change in $\phi_{N}$ is thought to be within the measizrement capabilities of current neutron diagnostics [10].
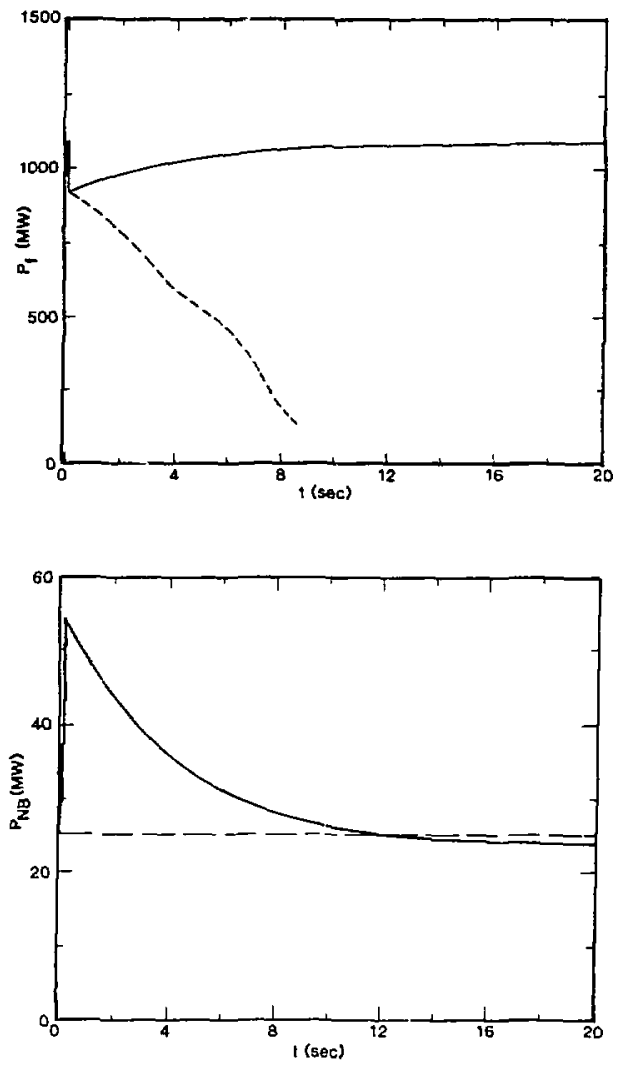

Figure 3: Simulations of an TTER thermal instability due to $\Delta T_{c, i}=-0.38 \mathrm{KeV}$ (or $\Delta \phi_{N} / \phi_{N}=-15 \%$ ) perturbation of the equilibrium point labeled with a - in Fig. 1. Shown are plots of (a) fusion power $P_{f}$ and (b) neutral beam power $P_{N B}$ as function of time for cases with (solid line) and without (dashed line) feedback. 


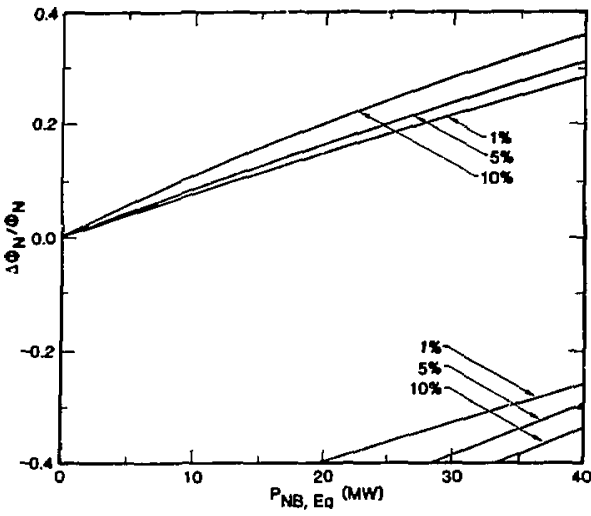

Figure 4: Plots of critical positive and negative initial perturbations $\Delta \phi_{N} / \phi_{N}$ as a function of $P_{N B, E q}$. Shown are curves for $c_{\alpha}=1,5$, and $10 \%$.

We see from Figs. 2 and 3 (solid lines) that applying neutral beam feedback as described by Eq. (5) readily stabilizes perturbations that resulted in disruptions (see dashed lines) without the feedback. In the case of both positive and negative excursions, equilibrium conditions are largely restored within $10 \mathrm{sec}$.

The equilibrium neutral beam power $P_{N E, E q}$ is a critical parameter in our control scheme and must be chosen with some care. Ideally, one would like to choose the smallest $P_{N B, E q}$ possible since that maximizes the nominal $Q$ value for the reactor. On the other hand, decreasing $P_{N B, E q}$ reduces the magnitudes of the initial positive perturbations that can be stabilized. This tradeoff is illustrated in Fig. 4 which describes simulations for a number of equilibrium points characterized by different $P_{N B, E_{q}}$ and $c_{\alpha}$. At each point, the ion and electron temperature were instantaneously perturbed by an amount sufficient to produce a given $\Delta \phi_{N} / \phi_{N}$ and the plasma was allowed to evolve in the presence of feedback described by Eq. (5). The initial perturbation was varied to yield the critical positive and negative $\Delta \phi_{N} / \phi_{N}$ values that could barely be stabilized. The curves in Fig. 4 are these critical perturbations.

We notice frum Fig. 4 that increasing $P_{N B, E q}$ increases the magnitude of the stabilizable positive perturbations but decreases the magnitude of the stabilizable negative perturbations. This occurs because of the limit on $P_{N B, m a x}$. Furthermore, we see that the minimum allowable choice for $P_{N B, E q}$ is primarily limited (for a particular $c_{\alpha}$ ) by the minimum positive $\Delta \phi_{N} / \phi_{N}$ value to be stabilizeci. This, in turn, is set by neutron diagnostic capabilities which we assume are no better than $\Delta \phi_{N} / \phi_{N}= \pm 15 \%[10]$. The minimum $P_{N B, E q}$ is also seen to decrease as $c_{\alpha}$ increases. However, recognizing that stability must be ensured both at the start of burn (when $c_{\alpha}=1 \%$ ) and at the end of burn (when $c_{\alpha}=10 \%$ ), we choose $P_{N B, E q}$ based on the $c_{\mathfrak{a}}=1 \%$ curve. This gives $P_{N B, E q}=21 \mathrm{MW}$ corresponding to $Q \approx 54$.

Based upon this analysis, we conclude that high- $Q$ IIER operation consistent with MFD stability, divertor, and fueling requirements might be possible at high density, low temperature equilibrium points if a neutral beam feedback system based on neutron flux measurements is employed to control thermal instabilities. Calculations with more complete plasma models will be undertaken to confirm this.

\section{Acknowledgements}

This work was supported by the Magnetic Fusion Energy Postdoctoral Research Program which is managed for the U.S. DOE by Oak Ridge Associated Universities; and also by Lawrence Livermore National Laboratory under the auspices of the U.S. DOE under contract W7405-ENG-48.

\section{References}

[1] The ITER Participants, International Thermonuclear Experimental Reactor (ITER). Establishment of ITER: Relevant Documents. Vienna: International Atomic Energy Agency, 1988, pp. 40-44.

[2] N.A. Uckan and the ITER Physics Group, "ITER Physies Design Guidelines," ITER-TN-PH-8-6, 1988.

[3] P. Yushmanov, T. Takizuka, K. Riedel, O. Kardaun, J. Cordey, and S. Kaye, "Tokamak Energy Scaling Expressions and Their Uncertainty," submitted to Nucl. Fusion, 1989.

[4] W.A. Houlberg, S.E. Attenberger, and L.M. Hively, "Contour Analysis of Fusion Reactor Plasma Performance," Nucl.Fusion, vol. 22, pp. 935-945. July 1982.

[5] L.J. Perkins, S. Putvinski, W. Spears, J. Galambos, "Physics Phase Parameters," ITER Technical Note ITER-IL-SA-1-9-27, 1989.

[6] S.K. Ho and M.E. Fenstermacher, "Thermally Stable Operation of Engineering Tesi Reactor Tokamaks," Fusion Technol. vol. 16, pp. 185-196, September 1989.

[7] L. Bromberg, J.L. Fisher, and D.R. Cohn, "Active Bura Control of Nearly lgnited Plasmas," Nucl. Fusion, vol. 20, pp. 203-207, Feiruary 1980.

(8] E.A. Chaniotakis, J.P. Freidberg, and D.R. Cohn, "Burn Control with Tailored Auxiliary Heating," presersted at 30th Annual Meeting of the Plasma Physics Division of the American Physical Society, Hollywood, FL, October 31-November 4, 1988.

(9] J. Mandrekas and W.M. Stacey, "Evaluation of Different Burn Contrul Methods for ITER," to be published in the Proceedings of the IEEE Symposium on Fusion Engineering, 1989.

[10] T. Elevant, "Requirements on Diagnostic. for Btirn Ccntrol," presented at the ITER Physics Group Meeting on Burn Control, Garching, West Germany, August, 1989. 


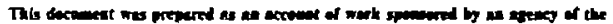

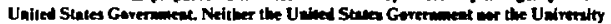

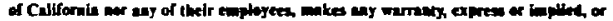

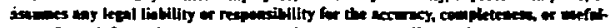

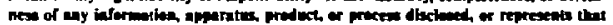

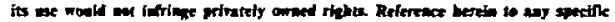

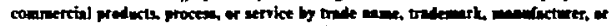

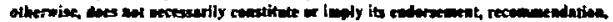

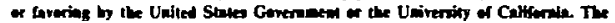

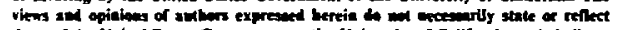

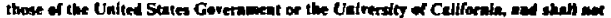
be wed for strertbing or preted endersenest proves.

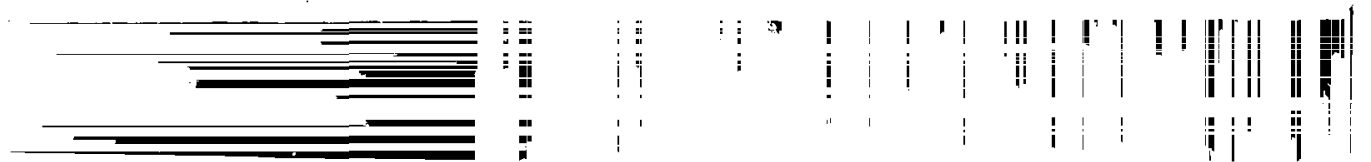

